author(s) Bruno Azevedo brunomiguelam@gmail.com University Polytechnic of València

Rute Bastardo rutebastardo@gmail.com

University of Trás-os-Montes and Alto Douro

Rubén Tortosa rtortosa@dib.upv.es

University Polytechnic of València

José Bártolo bartolo.jose@gmail.com

School of Art and Design

\title{
Information Visualization: An analysis On The Data Glut And The Emergency To Rethink And Design New Communicative Paradigms
}

abstract This article aims to relate the current condition of contemporary society, the noninformation society that is characterized by a ubiquitously multidirectional information flow, and an urgent need to induce reflection, intervention and repositioning by the Design discipline about this problematic. Given the increased difficulty in effectively communicating information in various sectors and services of our society, an efficient communication of data is a contradictory fact that the global networked society faces. In this sense, this article demonstrates that, designers are faced with the urgent task to rethink the existing processes of communication and information systems, but also develop new tools in order to search, filter, framework and efficiently visualize complex amounts of data. The task of understanding, conceptualizing and designing our complex information systems in a comprehensible and accessible manner, currently represents an important goal and a vast field of action.

This paper shows the need to deepen the study about the emergent subject that is the field of Information Visualization. The work here presented is a starting point discussion on the subject, which aims to find a solution to the problem of the non-information contemporary society, focusing specifically on the importance of Information Visualization, in order to provide further reflections on the topic. data glut, organization, information visualization design

\section{Introduction}

The decoding of patterns and the viewing of significant evidence in large amounts of data represents one of the greatest challenges of the 21st century. Compiling, organizing and viewing information has been a task of humanity for thousands of years. Repositories of knowledge such as files, maps, encyclopedias, libraries and current databases show the cultural evolution of information systems (Wright 2008). The amount of information produced and made available on the Web has reached an unprecedented scale, which metaphorically represents the exponential dimension of information. In fact, the problem of the organization, classification and framework of information is an important issue. It is common to several sectors, from large institutions and organizations to the average user who downloads, shares and stores data on his/her computer. All have come across and faced difficulties in classifying and organizing the multiple types of stored files, as well as what concerns to the quantification, description, organization and interpretation of large 
volumes of data.

The Era of information has brought forth profound changes in social daily life, making it essential to recognize and assimilate that the principles governing the disclosure of information are changing. The issues inherent to the efficient communication of information are a contradictory fact of the current global network society. A typical example of this is that there is still a great difficulty in effectively communicating information in various sectors of society and services (Wurman 2001: 9).

It is therefore, considered that this issue needs attention and action fro $m$ the Design/ er, with the aim of developing for searching, filtering and visualize a complex volume of information. Wurman (2001: 9), states that the task of developing and exploiting new forms that aim for a more efficient meaning of content is entirely the responsibility of the Design/er. According to Schuller (2009: 3), Designers, Researchers and Information Architects (Wurman 2001: 23), establish standards for classifying and organizing complex repositories and databases, which in turn influence the way knowledge is communicated and visualized (Schuller 2009: 3). The task of designing our systems of information in an understandable and accessible way is an important goal, taking into account the contemporary challenge of overload and congestion prompted by non-information.

\section{Information Glut}

Information as "matter" (Ciuccarelli 2009), has been over the times a valuable resource for biological, historical and cultural evolution. The Information Age doesn't have its ancestry in Gutenberg's movable type press or the development of the microchip, as it has been wrongfully attributed, but, according to Wright (2008: 12), in complex biological evolution of early life forms. In each new Era, we have witnessed an exponential growth of information, partly as a result of the democratization of access and production of information. The development of movable typed press, the telegraph, the typewriter, the telephone, radio, computer and internet, each referring to his time, became apparent a new complexity in human communication characterized by the abundance of information (Gleick 2011: 398). The boom of printed data, and the increasing volume of data, forcibly pushed up to today the development of systems and methods to organize information (Wright 2008: 121).

According to Wurman (2001: i), what in fact this reflects is not an excess of information, but an explosion of "non-information", or simply data that tell you nothing. Accordingly, the user is characterized as a "prosumer " for he plays two roles simultaneously: as a consumer but also as a content producer (ibid. 2001: 8). Consequently, the abundance of information is presented simultaneously as a disaster, because ninety-nine percent of the data are not meaningful or understandable; an opportunity because of the exponential volume of information generated (ibid. 2001: 9), which thus opens an endless field of activity contexts. The current processing capacity of computers, and the development of storage at relatively low cost, lead companies, institutions and organizations to manipulate and store large volumes of data. This increased the problems related to the organization, and communication of information (idem. 2001: 9). This problem assumes a role of dominance increasingly significant, in supporting decision-making and understanding of complex patterns and evidence (Lima 2011: 11) imbued "imperceptibly "in the data. Indeed, increased storage capacity and data publication led to a relentless pursuit of information sources based on their own interests (Wurman 2001: 8). This, in turn, allowed independent access to a type of information that was not previously available. Nowadays, the user has at his disposal several tools that allow a capacity of more rigorous and 
sustained research, taking into account the pros and cons of a decision, especially when purchasing a particular product, such as a book or an application. A typical example is that we often search for comparisons, reviews and comments within a network community with a common interest on a specific product or content. This fact highlights the need to rethink the way information is communicated and organized, as research around a specific content is often subject to a process of filtering and sorting (ibid. 2001: 9) by users. In earlier times there was noticeably a huge effort from civilization in order to store and collect information (Wright 2008: 48-58). Currently, is evidenced the adoption of strategies that maximize a reduction in the volume of information (Wurman 2001: 9).

The abundance of data reveals a large dimension problem in terms of organization and credibility (ibid. 2001: 6). This finding implies the perception that credible information is at the same level of non-credible information, since both are shared and disseminated at the same level (ibid. 2001: 13). However, the intrinsic problem of the volume of data is not in the issue of the amount of information itself. The main question ensues during the process of knowledge, namely to effectively filter and frame the information in the user's cognitive and perceptual field. Thus, it is imperative to rethink the current information systems but also the development of new communicative paradigms.

\section{Rethinking New Communicative Paradigms}

Faced with an abundance of data, we witness the development of new industries in the field of data management and analysis (eg, data mining), which transform data into useful information (ibid. 2001: 2). However, the need to structure an interactive, efficient and functional relationship with a large data set, has become an important objective in the current paradigm of the abundance of non-information society. In fact, we are flooded, either cognitively or perceptually, by a type of information that does not match our specific interest (ibid. 2001: 14-15), for a large part of the published and accessed daily information is not subject to a process of credible and efficient filtration (Thackara 2006: 163). This process should consider not only the present state of knowledge (Fig.1), but also the shape, structure and framework as fundamental aspects in the relationship between the user and the data (idem 2006: 163).

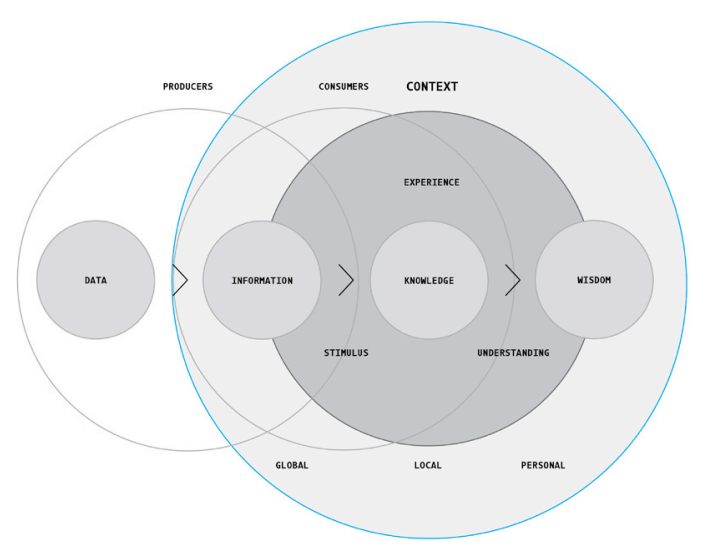

Figure 1. Nathan Shedroff (1994), The Continuum of Understanding 
These principles are corroborated by Ciuccarelli (2009), when he stresses the need to give this new "matter" a form, a structure and a framework in the user's cognitive and perceptual field. In this sense, Wurman (2001: 10), highlights that the organization of content is as important as the content itself. Refine, search, organize and visualize information, constitutes therefore one of the current main priorities (idem 2001: 10). In fact, Information Visualization is the process of analyzing and converting data into graphical representations, and it acquires great importance as it brings together several different disciplines (Fry 2008: 5). It enables to go far beyond simple data gathering as it allows viewing in an analytic and synthetic way. In this sense, Information Visualization is a new "medium", a new discipline that has as goals the description, representation, organization and quantification of data into visual images. This shares common characteristics and principles to information visualization techniques that digitally emerged in the early ' 90 , like the graphs used in digital apps (Manovich in Lima 2011: 11). However, what distinguishes this course from traditional visualization techniques is the interactivity component. For, on the one hand the graphical representations enable, perceptually and cognitively, the transformation of large and complex volumes of data into something visually accessible, Information Visualization incorporates interactivity, (Fry 2008: 5), thereby allowing an adaptation of representations and a maximization of the process inherent to the research and data visualization.

\section{Conclusion}

The information abundance problematic is directly related with the fact that, for the majority of information to which we are exposed, a process of filtering, classification and contextualization has not yet been applied. This demonstrates the urgency in developing strategies that prioritize the search and organization tasks, improving the way information is stored, framed and presented (Wurman 2001: 10).

Regarding to future lines of research, it is necessary to briefly explain a problem that emerges from the relationship of the user with a repository of information, (for example a Library, or an Academic Scientific Repository). The following example illustrates metaphorically the referenced problem: when we stand before a large amount of data, and according to our particular subject, we drove to a specific section, it is frequent we came across with a vast informational ocean (Wright 2008: 171-175). In this sense, the question that arises from this experience is logically what is the most appropriate or specific information, taking into account the theme/subject or topic of our specific research. However, if we think that this information is accessed by a significant number of users with a specific interest in a theme, subject, topic, author, typology, date of publication, and in the course of the research, they handle a significant amount of information, it is then possible to consider the existence of a relational structure of evidences, as a result of the relationship between the various users and their specific interests.

According to the problem and specific context described, namely the relationship between the user and an Academic repository, as the Open Academic Repository of the University of Porto, RepositóriUM (University of Minho), or RiuNet UPV (Institutional Repository of the University Polytechnic of Valencia), they only allow the statistically view of the number of times that the information was consulted (RepositóriUM), or specify a distribution by typology (eg. by author, key words, area of knowledge) in RiuNet. In the case of the interface used in RiuNet it is also possible to search by relevance, popularity and assign comments. However, even when this data is available, it is not possible to understand the pertinence and relevance of the information for the users, in other words, visualize the relational structure that emerges from the interaction of users with 
the queried information, as in the case of the MACE project (Metadata for Architectural Contents in Europe) of Stefaner (2009) (Fig. 2).

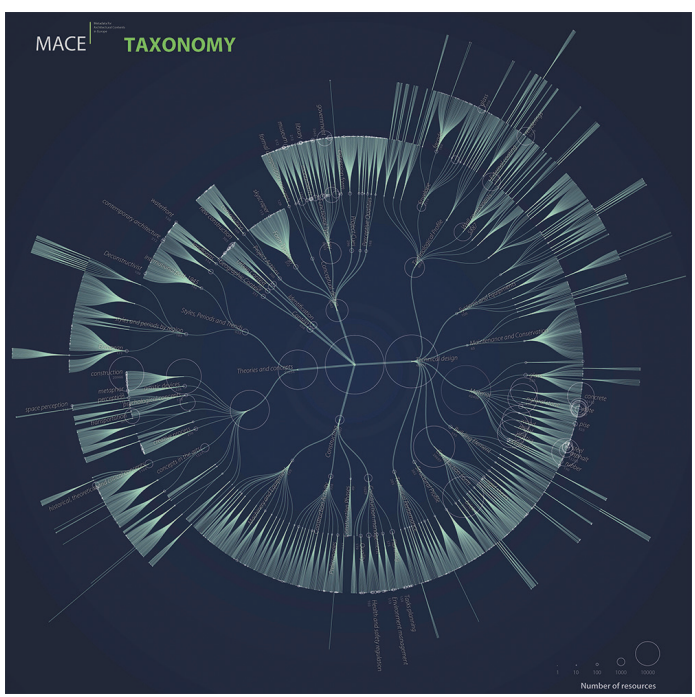

Figure 2.

Moritz Stefaner (2006), Mace.

In this sense, it is essential to visualize the relational structure that emerges between the user and its research, and present this information to the community, so that the individual feedback of each user contribute to an overall more efficient search.

This implies that the user of the open networked communities shares "what he knows and how he feel", creating a "database" liable to extract knowledge and create opportunities. (Smith cit. in Rheingold 2002: 30). However, as emphasized by Dellarocas (cit. in Rheingold 2002: 127), the main problem detected in open systems/reputation technologies, lies in the vulnerability associated with the manipulation of ratings.

Thus, the proposal to solve the problem stated, results from the conceptualization and study of a collaborative interface for the classification of data, allowing the user to view the structure, which comes from the social relationship that is established between the community and the search for information. In this sense, the goal is to interpret, summarize and present dynamically and interactively (in real time) the emerging relational structure of evidences, resulting from the connections concerning to user interaction with the search and retrieval of information. Therefore instead of the usual "object" centred approach, an approach based on the user experience will be established.

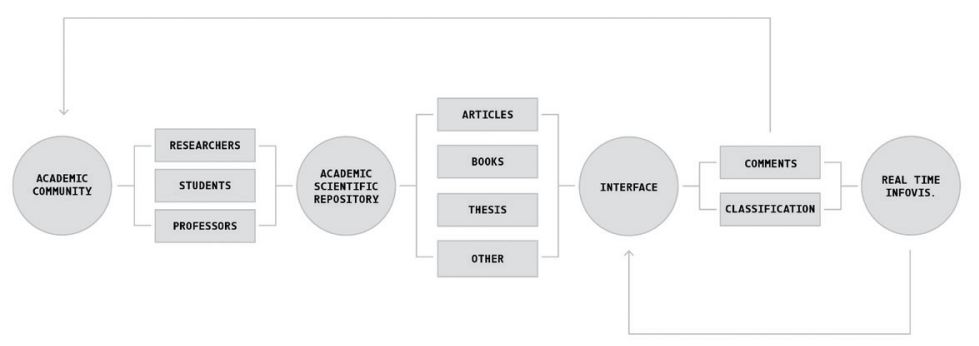

Figure 3 .

Interface Architecture 
It aims to present results, which comes from the relationship between an "close" networked community and the contents searched, based on an interaction of cooperative support for the classification and content enrichment (metadata) (Fig. 3). In this sense, the classification process unifies an interactive networking "dialogue" between a "close" networked community and the information.This paper aims to highlight the relationship of a problem concerning the search and filtering of information. It also highlights the importance to visualize the relational structure of evidences, that emerges from the relationship established between the user and the data querying. In this way, and according to the referenced context, the present reflection should be stimulated in the area. This is the main objective in the present work, in order to edify as contribution, the reflection over a problematic phenomena that already indicates a decisive transformation inside the discipline and the way of designing, through an emerging field.

\section{References}

Chen, C. (2006) Information Visualization: Beyond the Horizon, and and edition, London: Springer-Verlag.

Ciuccarelli, P. (2009), 'Living with Information: Architecture and Visualization' (video file). [Electronic], Available from: < http://www.vimeo.com/8012824> [22 December 2012]. Fry, B. (2008) Visualizing Data, and 1st edition, Sebastopol: O'Reilly Media, Inc. Gleick, J. (2011) The Information, A History, A Theory, A Flood, New York: Vintage Books.

Lima, M. (2011) Visual Complexity: Mapping Patterns of Information, New York: Princeton Architectural Press.

Moritz, S. (2009), 'Living with Information: Architecture and Visualization' (video file).

[Electronic], Available from: <http://vimeo.com/8012824> [22 December 2012]. Rheingold, H. (2002) Smart Mobs The Next Social Revolution, USA: Perseus Basic Books. Schuller, G. (2009) The World as Flatland Report 1: Designing Universal Knowledge, Baden: Lars Müller Publishers.

Shedroff, N. (1994), 'Information Interaction Design A Unified Field Theory of Design'. [Electronic], Available from: <http://www.nathan.com/thoughts/unified/unified.pdf> [01 August 2013]. Thackara, J. (2006) In the Bubble: Designing in a Complex World, London: MIT Press. Wright, A. (2008) Glut Mastering Information Through the Ages, Ithaca and London:

Cornell University Press.

Wurman, R. (2001) Information Anxiety 2, Indiana: QUE. 